\title{
AN APPROACH TO SOLVING DISCRETE VECTOR OPTIMIZATION PROBLEMS \\ OVER A COMBINATORIAL SET OF PERMUTATIONS ${ }^{1}$
}

\begin{abstract}
Complex discrete multicriteria problems over a combinatorial set of permutations are analyzed. Some properties of an admissible domain for a combinatorial multicriteria problem embedded into an arithmetic Euclidian space are considered. Optimality conditions are obtained for different types of effective solutions. A new approach to solving the problems formulated is constructed and substantiated.
\end{abstract}

Keywords: multi-objective optimization, combinatorial set, set of permutations, Pareto-optimal solution.

\section{INTRODUCTION}

At the present time, publications of many foreign and domestic scientists are devoted to the construction of efficient methods for solution and investigation of various aspects of the theory of multicriteria optimization problems including discrete ones [1-8].

Interest in the investigation of discrete multicriteria optimization models is conditioned by their wide application for solving important problems of economy, designing complicated systems, making decisions under uncertainty, etc. In solving various economic problems, including designing and distributing objects, planning experiments, and controlling data processing processes, various optimization problems over combinatorial sets play an important role. To date, essential results are obtained in the field of investigation of various classes of combinatorial models and development of new methods of their solution.

As is well known, the majority of combinatorial optimization problems can be reduced to problems of integer programming, but this is not always justified since the possibility of taking into account combinatorial properties of a problem [2] is lost in this case.

As is shown in [9-11], the convex envelope of a set of permutations is a common permutable polyhedron whose set of vertices is equal to the set of permutations. This property of a permutable polyhedron allows one to reduce the solution of a problem defined on a discrete combinatorial set to the solution of the problem over a continuous feasible set.

Based on the interrelation (established by the authors) between multicriteria problems over combinatorial sets and optimization problems over a continuous feasible set, the possibility arises to apply classical methods of continuous optimization to the solution of vector combinatorial problems over various combinatorial sets and also to develop new original solution methods using properties of combinatorial sets and their convex envelopes.

At present, there are many methods of solution of multicriteria problems, but none of them can be applied (in its general form) to combinatorial problems over permutations and, hence, the development of special methods and approaches to the solution of multicriteria problems over a combinatorial set of permutations is sufficiently important.

In this article, some characteristics of the construction of an admissible domain are established and also several theorems on the properties of different forms of efficient solutions of the problems being considered are formulated and proved. A possible approach is proposed to the solution of vector combinatorial-type problems over permutations.

\footnotetext{
${ }^{1}$ This work was supported by the Fundamental Research Fund of Ukraine (project $\left.\Phi 251 / 094\right)$.
}

Cybernetics Institute, National Academy of Sciences of Ukraine, Kiev, Ukraine, nvsemenova@meta.ua; ludapl@ukr.net; vpn2006@rambler.ru. Translated from Kibernetika i Sistemnyi Analiz, No. 3, pp. 158-172, May-June 2008. Original article submitted January 11, 2008. 


\section{PROBLEM STATEMENT. BASIC CONCEPTS AND DEFINITIONS}

To state the problem, we use the concept of a multiset $A$ that is specified by its base $S(A)$ and the multiplicity of its elements $k(a)$ [9-11].

Let a multiset $A=\left\{a_{1}, a_{2}, \ldots, a_{q}\right\}$, its base $S(A)=\left\{e_{1}, e_{2}, \ldots, e_{k}\right\}$, where $e_{j} \in R^{1} \forall j \in N_{k}=\{1, \ldots, k\}$, and the multiplicity of elements $k\left(e_{j}\right)=r_{j}, j \in N_{k}, r_{1}+r_{2}+\ldots+r_{k}=q$, be given.

By an ordered $n$-sample from the multiset $A$ we understand a collection

$$
a=\left(a_{i_{1}}, a_{i_{2}}, \ldots, a_{i_{n}}\right),
$$

where $a_{i_{j}} \in A \quad \forall i_{j} \in N_{n}, \forall j \in N_{n}, i_{s} \neq i_{t}$, if $s \neq t \quad \forall s \in N_{n}, \forall t \in N_{n}$.

Definition 1 [9-11]. A set $E(A)$ whose elements are $n$-samples of the form (1) from the multiset $A$ is called an Euclidean combinatorial set if, for its arbitrary elements $a^{\prime}=\left(a_{1}^{\prime}, a_{2}^{\prime}, \ldots, a_{n}^{\prime}\right)$ and $a^{\prime \prime}=\left(a_{1}^{\prime \prime}, a_{2}^{\prime \prime}, \ldots, a_{n}^{\prime \prime}\right)$, the conditions $\left(a^{\prime} \neq a^{\prime \prime}\right) \Leftrightarrow\left(\exists j \in N_{n}: a_{j}^{\prime} \neq a_{j}^{\prime \prime}\right)$ are satisfied. In other words, two elements of the set $E(A)$ are different from one another if, in addition to other distinctions, they have different arrangements of symbols that form them.

A set $P_{n k}(A)$ of permutations with repetitions among $n$ real numbers including $k$ different numbers is called a general set of permutations, i.e., a set of ordered $n$-samples of the form (1) from the multiset $A$ under the condition $n=q>k$.

When $n=k=q$, we have a set of permutations without repetitions. We denote it by $P_{n}$. It is obvious that we have $P_{n}(A)=P_{n n}(A)$. We denote by $P(A)$ a set of permutations in the case when the kind of permutations is not concretely specified. Let us consider multicriteria combinatorial problems of the form

$$
Z\left(\Phi, P_{n k}(A)\right): \max \left\{\Phi(a) \mid a \in P_{n k}(A)\right\}
$$

that consist of maximizing a vector criterion $\Phi(a)$ on an Euclidean combinatorial set of permutations $P_{n k}(A)$, where $\Phi(a)=\left(\Phi_{1}(a), \Phi_{2}(a), \ldots \ldots, \Phi_{l}(a)\right), \Phi_{i}: E(A) \rightarrow R^{1}, i \in N_{l}$.

There are many different principles of decision-making in such situations. Let us consider some of the most traditional principles connected with the selection of sets of vectors that are Pareto unimproved or optimal, Slater optimal, and Smale optimal from the entire set $Y=\left\{y=\Phi(a) \mid a \in P_{n k}(a)\right\}$. Thus, by a solution of a problem $Z\left(\Phi, P_{n k}(A)\right)$, we understand the determination of elements of one of the following sets: the set of Pareto-optimal (efficient) solutions $\mathrm{P}\left(\Phi, P_{n k}(A)\right)$, the set of Slater optimal (weakly efficient) solutions $\mathrm{Sl}\left(\Phi, P_{n k}(A)\right)$, and the set of Smale optimal (strictly efficient) solutions $\operatorname{Sm}\left(\Phi, P_{n k}(A)\right)$. We denote the problems of searching for elements from the sets $\mathrm{P}\left(\Phi, P_{n k}(A)\right)$, $\mathrm{Sl}\left(\Phi, P_{n k}(A)\right)$, or $\operatorname{Sm}\left(\Phi, P_{n k}(A)\right)$ by $Z_{\mathrm{P}}\left(\Phi, P_{n k}(A)\right), Z_{\mathrm{Sl}}\left(\Phi, P_{n k}(A)\right)$, and $Z_{\mathrm{Sm}}\left(\Phi, P_{n k}(A)\right)$, respectively. According to [3, 7, 8], for any $a \in P_{n k}(A)$, the following statements are true:

$$
\begin{gathered}
a \in \mathrm{Sl}\left(\Phi, P_{n k}(A)\right) \Leftrightarrow\left\{y \in P_{n k}(A) \mid \Phi(y)>\Phi(a)\right\}=\varnothing, \\
a \in \mathrm{P}\left(\Phi, P_{n k}(A)\right) \Leftrightarrow\left\{y \in P_{n k}(A) \mid \Phi(y) \geq \Phi(a), \Phi(y) \neq \Phi(a)\right\}=\varnothing, \\
a \in \operatorname{Sm}\left(\Phi, P_{n k}(A)\right) \Leftrightarrow\left\{y \in P_{n k}(A) \mid \Phi(y) \geq \Phi(a), y \neq a\right\}=\varnothing .
\end{gathered}
$$

It is obvious that we have

$$
\operatorname{Sm}\left(\Phi, P_{n k}(A)\right) \subset \mathrm{P}\left(\Phi, P_{n k}(A)\right) \subset \mathrm{Sl}\left(\Phi, P_{n k}(A)\right) .
$$

The finiteness of the admissible domain of permutations $P_{n k}(A)$ provides the nonemptiness of the set $\mathrm{P}\left(\Phi, P_{n k}(A)\right)$ and its external stability [8], $\forall y \in P_{n k}(A) \exists a \in \mathrm{P}\left(\Phi, P_{n k}(A)\right): \Phi(a) \geq \Phi(y)$. In the case of an infinite set $A$, the question of the existence of elements of the set $\mathrm{P}\left(\Phi, P_{n k}(A)\right)$ requires a special investigation.

\section{PROPERTIES OF A SET OF FEASIBLE SOLUTIONS}

We will consider elements of a set of permutations with repetitions as points of an arithmetic Euclidean space $R^{n}$.

Let a vector $a$ of the form (1) be an element of an Euclidean combinatorial set $E(A)$. A mapping $\varphi: E(A) \rightarrow E_{\varphi}(A) \subset R^{n}$ is called an embedding of the set $E(A)$ into the arithmetic Euclidean space if $\varphi$ specifies a one-one mapping $E_{\varphi}(A) \subset R^{n}$ according to the following rule: for $a=\left(a_{i_{1}}, \ldots, a_{i_{n}}\right) \in E(A), x=\varphi(a), x=\left(x_{1}, \ldots, x_{n}\right) \in E_{\varphi}(A)$, we have $x_{j}=a_{i_{j}} \quad \forall j \in N_{n}$. 
As is shown in $[9,10]$, the convex envelope of a set of permutations is a permutable polyhedron $\Pi_{n k}(A)=$ conv $P_{n k}(A)$ whose set of vertices is equal to the set $P_{n k}(A)$ of permutations, vert $\Pi_{n k}(A)=P_{n k}(A)$.

Without loss of generality, we order the elements of the multiset $A$ in nondecreasing order,

$$
a_{1} \leq a_{2} \leq \ldots \leq a_{n},
$$

and the elements of its base in increasing order, $e_{1}<e_{2}<\ldots<e_{k}$. Then the convex envelope of the general set of permutations $P_{n k}(A)$ is a general permutable polyhedron $\Pi_{n k}(A)$ that is described by the following system of linear inequalities:

$$
\left\{\begin{array}{l}
\sum_{j=1}^{n} x_{j} \leq \sum_{j=1}^{n} a_{j}, \\
\sum_{j=1}^{i} x_{\alpha_{j}} \geq \sum_{j=1}^{i} a_{j},
\end{array}\right.
$$

$\alpha_{j} \in N_{n}, \alpha_{j} \neq a_{t} \forall j \neq t, \forall j, t \in N_{i}, \forall i \in N_{n-1}$, and $P_{n k}(A)=\operatorname{vert} \Pi_{n k}(A)$.

The system of constraints (7), (8) of the general permutable polyhedron $\Pi_{n k}(A)$ can be written in the form of the following equivalent system:

$$
\left\{\begin{array}{l}
\sum_{i \in \omega_{t}} x_{i} \geq \sum_{i=1}^{\left|\omega_{t}\right|} a_{i} \quad \forall \omega_{t} \subset N_{n} \\
\sum_{i=1}^{n} x_{i}=\sum_{i=1}^{n} a_{i},
\end{array}\right.
$$

where $\left|\omega_{t}\right|$ is the number of elements of the set of indices $\omega$ defined as $\left|\omega_{t}\right|=t, t \in\{1,2, \ldots\}$.

In mapping a set of permutations $P_{n k}(A)$ into an Euclidean space $R^{n}$, we can formulate the problem $Z(F, X)$ of maximization of some vector criterion $F(x)$ on a set $X$; in this case, to each point $a \in P_{n k}(A)$ corresponds a point $x \in X$ such that we have $F(x)=\Phi(a)$ and

$$
Z(F, X): \max \{F(x) \mid x \in X\},
$$

where $F(x)=\left(f_{1}(x), f_{2}(x), \ldots, f_{l}(x)\right), f_{i}(x)$ corresponds to a functional $\Phi_{i}(a), f_{i}: R^{n} \rightarrow R^{1}, i \in N_{l}$, and $X$ is a nonempty set that belongs to $R^{n}$ and is defined as follows: $X=\operatorname{vert} \Pi_{n k}(A), \Pi_{n k}(A)=\operatorname{conv} P_{n k}(A)$. By the correspondence of a vector function $F$ to a vector of functionals $\Phi$ we understand the relationship $\Phi(a)=F(\varphi(a)) \forall a \in P_{n k}(A)$.

The problem $Z(F, X)$ can also contain additional linear constraints forming a convex polyhedral set $D \subset R^{n}$ of the form $D=\left\{x \in R^{n} \mid B x \leq d\right\}$, where $d \in R^{m}$ and $B \in R^{m \times n}$. Thus, the feasible set is of the form

$$
X=\operatorname{vert} \Pi_{n k}(A) \cap D .
$$

As a rule, the introduction of additional constraints complicates the problem but reduces the admissible domain, which should be taken into account in constructing solutions.

\section{OPTIMALITY CONDITIONS AND SOME PROPERTIES OF SETS OF EFFICIENT SOLUTIONS}

We denote the solution sets of the problem $Z(F, X)$ as follows: $\mathrm{P}(F, X)$ is the set of Pareto-optimal (efficient) solutions, $\mathrm{Sl}(F, X)$ is the set of Slater optimal (weakly efficient) solutions, and $\operatorname{Sm}(F, X)$ is the set of Smale optimal (strictly efficient) solutions. For any $x \in X$, the following statements that are of the form (2)-(5) and are written as applied to the problem $Z(F, X)$ are true:

$$
\begin{gathered}
x \in \operatorname{Sl}(F, X) \Leftrightarrow\{y \in X \mid F(y)>F(x)\}=\varnothing, \\
x \in \mathrm{P}(F, X) \Leftrightarrow\{y \in X \mid F(y) \geq F(x), F(y) \neq F(x)\}=\varnothing, \\
x \in \operatorname{Sm}(F, X) \Leftrightarrow\{y \in X \mid y \neq x, F(y) \geq F(x)\}=\varnothing, \\
\operatorname{Sm}(F, X) \subset \mathrm{P}(F, X) \subset \operatorname{Sl}(F, X) .
\end{gathered}
$$


Since the admissible domain $X$ is bounded, the set $\mathrm{P}(F, X)$ is not empty and is externally stable.

THEOREM 1. The elements of the sets $\operatorname{Sm}(F, X)$ of strictly efficient, $\mathrm{P}(F, X)$ of Pareto-optimal, and $\mathrm{Sl}(F, X)$ of weakly efficient solutions of a multicriteria combinatorial problem over permutations of the form $Z(F, X)$ are located at the vertices of a permutable polyhedron $\Pi_{n k}(A)$.

Proof. Taking into account relationships (14) between the introduced sets of efficient solutions and also according to $[9,10]$, the fact that the set of feasible solutions $X$ is a subset of the set of vertices of the general permutable polyhedron $\Pi_{n k}(A)$, i.e., $P_{n k}(A)=$ vert $\Pi_{n k}(A)$, we arrive at the conclusion that the chain of inclusions $\operatorname{Sm}(F, X) \subset \mathrm{P}(F, X) \subset$ $\mathrm{Sl}(F, X) \subset \operatorname{vert} \Pi_{n k}(A)$ takes place. The theorem is proved.

Let functions $f_{i}(x), i \in N_{l}$, of the vector criterion $F(x)$ be linear, i.e., we have $f_{i}(x)=\left\langle c_{i}, x\right\rangle, c_{i} \in R^{n}, i \in N_{l}$. The important properties of the admissible domain $X$, sets of efficient solutions of different kinds mentioned in Theorem 1, and also the linearity of functions of the vector criterion allow one to reduce the solution of the problem $Z(F, X)$ to the solution of the problem $Z(F, G)$ over the continuous feasible set $G=\Pi_{n k}(A) \cap D$.

Let $C \in R^{n \times l}$ be a matrix, and let $c_{i}$ be its row vector, $i \in N_{l}$. We represent the polyhedron $\Pi_{n k}(A)$ in the form $\Pi_{n k}(A)=\left\{x \in R^{n} \mid\left\langle\pi_{i}, x\right\rangle \leq \gamma_{i}, i \in N_{p}\right\}$ by reducing all the inequalities to one form $(\leq)$ and denote it by $\Pi$. We will analyze the problem $Z(F, X)$ taking into account the properties of the cone $K=\left\{x \in R^{n} \mid C x \geq 0\right\}$ of promising directions [3] of the problem $Z(F, X)$ and convex closed cones $0^{+} \Pi(y)=\left\{x \in R^{n} \mid\left\langle\pi_{i}, x\right\rangle \leq 0, i \in N(y)\right\}$, where $N(y)=\left\{i \in N_{p} \mid\left\langle\pi_{i}, y\right\rangle=\gamma_{i}\right\}$, that can be constructed for all points $y \in$ vert $\Pi$. It is obvious that we have $N(y) \neq \varnothing$ and $X \subseteq y+0^{+} \Pi(y)$. We denote by $K_{0}=\left\{x \in R^{n} \mid C x=0\right\}$ the kernel of a mapping $C: R^{n} \rightarrow R^{l}$ and by int $K=\left\{x \in R^{n} \mid C x>0\right\}$ the interior of the cone $K$. It follows from formulas (11-(13) that, $\forall x \in X$, the following statements are true:

$$
\begin{gathered}
x \in \mathrm{Sl}(C, X) \Leftrightarrow(x+\operatorname{int} K) \cap X=\varnothing, \\
x \in \mathrm{P}(C, X) \Leftrightarrow\left(x+K \backslash K_{0}\right) \cap X=\varnothing, \\
x \in \operatorname{Sm}(C, X) \Leftrightarrow(x+K) \cap X \backslash\{x\}=\varnothing .
\end{gathered}
$$

The theorems formulated below are true.

THEOREM 2. $\mathrm{P}(F, G) \cap$ vert $\Pi \subset \mathrm{P}(F, X), \mathrm{Sl}(F, G) \cap$ vert $\Pi \subset \mathrm{Sl}(F, X)$, and $\operatorname{Sm}(F, G) \cap$ vert $\Pi \subset \operatorname{Sm}(F, X)$.

Proof. Since we have vert $\Pi \cap D \subset G$, we obtain $\mathrm{P}(F, G) \cap$ vert $\Pi \cap D \subset \mathrm{P}(F, G \cap$ vert $\Pi \cap D)=\mathrm{P}(F, X)$. The relationships $\operatorname{Sl}(F, X)=\operatorname{Sl}(F$, vert $\Pi \cap D) \supset \operatorname{Sl}(F, G) \cap$ vert $\Pi \cap D$ and $\operatorname{Sm}(F, X)=\operatorname{Sm}(F, D \cap$ vert $\Pi) \supset \operatorname{Sm}(F, G)$ $\cap$ vert $\Pi$ are similarly proved.

THEOREM 3. If the feasible set $X$ of the problem $Z(F, X)$ does not contain constraints describing a convex polyhedral set $D$ or $\Pi \subseteq D$, i.e., we have $X=$ vert $\Pi$, then, $\forall x \in R^{n}$, the following statements are true:

$$
\begin{gathered}
x \in \mathrm{Sl}(F, X) \Leftrightarrow x \in \operatorname{Sl}(F, \Pi) \cap \operatorname{vert} \Pi, \quad x \in \mathrm{P}(F, X) \Leftrightarrow x \in \mathrm{P}(F, \Pi) \cap \operatorname{vert} \Pi, \\
x \in \operatorname{Sm}(F, X) \Leftrightarrow x \in \operatorname{Sm}(F, \Pi) \cap \operatorname{vert} \Pi .
\end{gathered}
$$

Proof. With allowance made for the conditions of Theorem 3, it follows from Theorem 2 that, $\forall x \in R^{n}$, the statements $x \in \mathrm{Sl}(F, \Pi) \cap$ vert $\Pi \Rightarrow x \in \mathrm{Sl}(F, X), x \in \mathrm{P}(F, \Pi) \cap$ vert $\Pi \Rightarrow x \in \mathrm{P}(F, X)$, and $x \in \operatorname{Sm}(F, \Pi) \cap$ vert $\Pi$ $\Rightarrow \operatorname{Sm}(F, X)$ are true. Let us prove the inverse implications. Let $x \in \operatorname{Sl}(F, X)$, whence it follows by Theorem 1 that we have $x \in$ vert $\Pi$. Proving by contradiction, we assume that $x \notin \mathrm{Sl}(F, \Pi)$. Taking into account the linearity of functions $f_{i}(x), i \in N_{l}$, of the vector criterion $F(x)$, we obtain by Theorem 1 from [5] that the condition int $K \cap 0^{+} \Pi(x) \neq \varnothing$ is true, i.e., some boundary points of the set $\Pi$ are within the cone $(x+\operatorname{int} K)$ and, hence, there is a point $x^{1} \in$ vert $\Pi$ belonging to this cone. By virtue of formula (12), the latter means that $x \notin \mathrm{Sl}(F, X)$ and contradicts the condition of the theorem. The other statements of this theorem are similarly proved. The proof is completed.

COROLLARY. Under the conditions of Theorem 3, the following equalities are true:

$$
\mathrm{P}(F, \Pi) \cap \operatorname{vert} \Pi=\mathrm{P}(F, X), \operatorname{Sl}(F, \Pi) \cap \operatorname{vert} \Pi=\operatorname{Sl}(F, X), \operatorname{Sm}(F, \Pi) \cap \operatorname{vert} \Pi=\operatorname{Sm}(F, X) .
$$

If the admissible domain of the problem $Z(F, X)$ is $X=\operatorname{vert} \Pi_{n k}(A)$, then, for any point $x \in \operatorname{vert} \Pi_{n k}(A)$, the necessary and sufficient optimality conditions obtained in [5] are true for all the above-mentioned kinds of efficient solutions.

If the admissible domain $X$ of the problem $Z(F, X)$ satisfies additional constraints specifying a convex polyhedron $D$, i.e., we have $X=$ vert $\Pi \cap D$ and $\Pi \cap D \neq \Pi$, then only sufficient optimality conditions of solutions are true. 
THEOREM 4. $\forall x \in$ vert $\Pi: x \in \mathrm{P}(F, \Pi) \cap D \Rightarrow x \in \mathrm{P}(F, X), x \in \operatorname{Sl}(F, \Pi) \cap D \Rightarrow x \in \operatorname{Sl}(F, X)$, and $x \in \operatorname{Sm}(F, \Pi)$ $\cap D \Rightarrow x \in \operatorname{Sm}(F, X)$.

Proof. Since $G=\Pi \mid D$, the following implication takes place $\forall x \in \operatorname{vert} \Pi: x \in \mathrm{P}(F, \Pi) \cap D \Rightarrow x \in \mathrm{P}(F$, $\Pi \cap D)=\mathrm{P}(F, G) \Rightarrow x \in P(F, X), x \in \mathrm{Sl}(F, \Pi) \cap D \Rightarrow x \in \mathrm{Sl}(F, X)$, and $x \in \operatorname{Sm}(F, \Pi) \cap D \Rightarrow x \in \operatorname{Sm}(F, X)$.

Thus, Theorems 1-4 establish an interrelation between the problem $Z(F, X)$ and the problem $Z(F, G)$ defined over a continuous feasible set. This allows one to apply classical continuous optimization methods to the solution of vector combinatorial problems over permutations and to develop new original solution methods on this basis, using properties of combinatorial sets and their convex envelopes.

In establishing different kinds of solution efficiency, one should take into account that if the necessary optimality conditions of a solution are satisfied, then its efficiency cannot be guaranteed but if these conditions are not satisfied, then the corresponding solution is inefficient. If sufficient conditions are used, then a solution that satisfies them is efficient and, otherwise, the question of efficiency of the solution remains open. But if necessary and sufficient conditions are used, then the question is uniquely solved, namely, a solution is efficient if and only if it satisfies these conditions.

If the problem $Z(F, X)$ does not contain linear constraints forming a convex polyhedral set $D \subset R^{n}$ or if we have $\Pi \subseteq D$, i.e., $X=$ vert $\Pi$, then, taking into account the necessary and sufficient optimality conditions (Theorem 3 ), the process of its solution is reduced to the search for efficient solutions of the problem $Z(F, G)$ over the continuous feasible set $G=\Pi$ with the subsequent choice of only the solutions that are vertices of the permutable polyhedron $\Pi$.

Analyzing Theorems 2 and 4, we obtain the following relationships between the problems $Z(F, X)$ and $Z(F, G)$ : if we have $x \in \mathrm{R}(F, G) \cap$ vert $\Pi$, then $x \in \mathrm{R}(F, X)$, and if we have $x \notin \mathrm{R}(F, G) \cap$ vert $\Pi$, then this does not imply that $x \notin \mathrm{R}(F, X)$, where $\mathrm{R}(F, X)$ denotes the set $\mathrm{P}(F, X), \operatorname{Sm}(F, X)$, or $\mathrm{Sl}(F, X)$.

\section{A GENERAL APPROACH TO THE SOLUTION OF VECTOR PROBLEMS OVER A COMBINATORIAL SET OF PERMUTATIONS}

If a problem $Z(F, X)$ contains additional linear constraints, then the following approach to its solution is proposed.

1. Find efficient solutions of the problem $Z(F, \Pi)$.

2. Check their membership in the set $D$. If we have $x \in \mathrm{P}(F, \Pi) \cap D$, then $x \in \mathrm{P}(F, X)$.

3. Consider feasible solutions $x \in X$ to the problem $Z(F, X)$ that are inefficient in the problem $Z(F, \Pi)$, i.e., $x \in X \backslash(\mathrm{P}(F, \Pi) \cap D)$, and check them for efficiency. To this end, use the necessary and sufficient conditions formulated in [8, pp. 187 and 188].

Statement 1. A feasible solution $x^{0}$ is efficient if and only if it is an optimal solution of the following problem:

$$
Z^{1}(f, X): \max \left\{\sum_{i=1}^{l} f_{i}(x) \mid x \in X, f_{i}(x) \geq f_{i}\left(x^{0}\right), i \in N_{l}\right\} \text {. }
$$

If the solution $x^{0}$ is inefficient, then, as a result of solution of this problem, we found an efficient solution $x^{*}$ that is more preferable than $x^{0}$, i.e., we have $F\left(x^{*}\right) \geq F\left(x^{0}\right)$.

Continuing investigations and developing the results of [1, 4-6,9-13], we propose an approach to the solution of the problem $Z(F, X)$ on the basis of linear convolution (aggregation) of its partial criteria and the further reduction of the search for solutions of the initial problem to the solution of a series of scalar (one-criterion) problems and the check of the obtained solutions for optimality. The method of solution of one-criterion problems is based on the ideas of decomposition, Kelly's cutting-planes, and relaxation. Next, we consider a method whose realization takes into account the fact that the number of constraints is sufficiently large. Then it is expedient to use a relaxation procedure or temporary rejection of some constraints and the solution of a problem over a wider domain, i.e., under remained constraints.

At the initial stage of construction of the sought-for algorithm, we should determine the initial point. We will consider a one-criterion problem without constraints that describe a polyhedron $D$ and call them additional constraints.

Statement 2. If, for the elements of the multiset $A$ and coefficients $c_{j}, j \in N_{n}$, of the objective function of the problem extr $\left\{f(x)=\sum_{j=1}^{n} c_{j} x_{j} \mid x \in \operatorname{vert} \Pi_{n k}(A)\right\}$, conditions (6) and $c_{i_{1}} \leq c_{i_{2}} \leq \ldots \leq c_{i_{n}}, i_{n} \in N_{n}$, respectively, are satisfied, then the maximum of the function $f(x)$ on the admissible set is attained at a point $x^{*}=\left(x_{i_{1}}^{*}, x_{i_{2}}^{*}, \ldots, x_{i_{n}}^{*}\right) \in$ vert $\Pi_{n k}(A)$ that is specified as follows:

$$
x_{i_{j}}^{*}=a_{j} \forall j \in N_{n},
$$


and its minimum is accordingly attained at a point $y=\left(y_{i_{1}}, y_{i_{2}}, \ldots, y_{i_{n}}\right)$, where

$$
y_{i_{j+1}}=a_{n-j} \forall j \in N_{n-1} \cup\{0\} \text {. }
$$

It should be noted that the total number $p$ of linear inequalities entering into system (7), (8) that describes a permutable polyhedron $\Pi_{n k}(A)$ is very large. According to [9-11], we call the set of inequalities of system (7), (8) that have the same value $i$ of the upper summation limit the $i$ th group of inequalities of this system. In particular, each $i$ th group contains $C_{n}^{i}$ inequalities. From this we obtain the total number of inequalities $p=\sum_{i=0}^{n} C_{n}^{i}=2^{n}$. Since only $k$ coordinates out of $n$ coordinates $a_{j}, j \in N_{n}$ are different, some inequalities can be eliminated from the system of inequalities (7), (8). Taking into account the truth of condition (6) for any $j \in N_{i-1}, i \leq n$, the equality $a_{j}=a_{j+1}$ is fulfilled. In this case, the truth of inequalities of the first group in system (7), (8) also implies the fulfillment of the inequalities of the second, third, .., $i_{n}$ th groups. In fact, since we have $x_{j} \geq a_{1}, j \in N_{n}$, for any $i \in N_{n}$, the condition $\sum_{j=1}^{i} x_{\alpha_{j}} \geq i a_{1}$ is true. Hence, from system (7), (8) describing a permutable polyhedron, we can eliminate the inequalities of the second, third, ..., ith groups and the total number of inequalities will be $N=1+n+\sum_{j=i+1}^{n} C_{n}^{j}$. If a collection of numbers $\left(a_{1}, a_{2}, \ldots, a_{n}\right)$ possesses a property $a_{j}=a_{j+1} \forall j \in N_{n-1} \backslash N_{n-i}$, then, in system (7), (8), it suffices to retain only the inequalities of the first, second, ..., $(n-j)$ th group.

At the first stage of solving a problem, it is important to correctly organize the choice of active inequality constraints. To solve the problem $Z(F, X)$ at the initial stage, it is necessary to take into account only the part of constraints that determine the domain $X$. Since the determination of efficient solutions of the problem $Z(F, X)$ is more important than the construction of the entire set of constraints describing the admissible domain $G$, it is sufficient to construct only the constraints of the set $G$ that determine efficient solutions of this problem. The method considered here is intended for obtaining such constraints.

We introduce the following denotations. We write the admissible domain of the problem $Z(F, G)$ in the form $G=\left\{x \in R^{n} \mid H x \leq g\right\}, g=\left(g_{1}, g_{2}, \ldots, g_{q}\right), H \in R^{q \times n}$; here, $H$ is a matrix used for the matrix-vector representation of constraints of the form (7), (8) and linear inequalities describing a polyhedron $D$, where all the constraints are reduced to one ( $\leq$ ) kind of inequalities. We denote by $N_{q}$ the set whose elements determine the numbers of constraints of system (7), (8) and additional constraints describing a convex polyhedral set $D: N_{q}=\left\{1,2, \ldots, 2^{n}+m\right\}$.

We define sets $G_{i}=\left\{x \in R^{n} \mid\left\langle h_{i}, x\right\rangle \leq g_{i}\right\}, i \in N_{q}$ and, for an arbitrary $x^{s} \in R^{n}$, define sets $N^{a}\left(x^{s}\right)=$ $\left\{i \in N_{q} \mid\left\langle h_{i}, x^{s}\right\rangle=g_{i}\right\}$ and $N^{n}\left(x^{s}\right)=\left\{j \in N_{q} \mid\left\langle h_{j}, x^{s}\right\rangle<g_{j}\right\}$ of active and inactive constraints, respectively, at the point $x^{s} ; h_{i} \in R^{n}, g_{i} \in R, i \in N_{q}$, are, respectively, the $i$ th row vector of the matrix $H$ and the $i$ th component of the vector $g$.

Let us consider the following problem:

$$
Z\left(F, G^{s}\right): \max \left\{F(x) \mid x \in G^{s}\right\}, \quad \text { where } G^{s}=\left\{x \in R^{n} \mid\left\langle h_{i}, x\right\rangle \leq g_{i}, i \in Q_{s} \subset N_{q}\right\}
$$

$Q_{S}$ is the set of indices of the constraints describing the admissible domain of the problem $Z\left(F, G^{S}\right)$ that is solved at the sth step of the algorithm, $Q_{S}=N_{q} \backslash R_{S}$, and $R_{S}$ is the set of numbers of the constraints that have not been included in this problem at the sth step.

Definition 2. We call the quantity $r_{i}(x)=\left\langle h_{i}, x\right\rangle-g_{i}, i \in N_{q}$, a deviation of a point $x \in R^{n}$ from the boundary of a set $G_{i}$ and the quantity $r(x)=\max \left\{r_{i}(x) \mid i \in N_{q}\right\}$ a deviation of a point $x \in R^{n}$ from the boundary of the set $G$.

It is obvious that, for $i \in N_{p}$, we have

$$
r_{i}(x)=\sum_{j=1}^{i} x_{\alpha_{j}}-\sum_{j=1}^{i} a_{j}
$$

and, for $i \in N_{q} \backslash N_{p}$, we have

$$
r_{i}(x)=\left\langle b_{i}, x\right\rangle-d_{i}
$$

where $b_{i}$ is the $i$ th row vector of the matrix $B, d_{i} \in R$. 
THEOREM 5. An efficient (Pareto-optimal, weakly efficient, and strictly efficient) solution $x_{0}$ of the problem $Z\left(F, G^{S}\right)$ is an efficient (in the same sense) solution of the problem $Z(F, G)$ if and only if the condition $r(x) \leq 0$ is true.

Proof. The necessity of this statement is obvious since the feasible solution $x_{0}$ of the problem $Z\left(F, G^{S}\right)$ is a feasible solution of the problem $Z(F, G)$ if and only if the condition $r(x) \leq 0$ is satisfied. The sufficiency of this statement follows from the construction of the problem $Z(F, G)$ and the definition of $r(x)$.

The main idea of the proposed method of solution of the problem $Z(F, X)$ is as follows. Put $s=0$.

1. Reduce the multicriteria problem $Z(F, G)$ to a one-criterion problem $Z(f, G)$ by the linear convolution method.

2. Choose the constraints of the initial system that determines a domain $G^{S} \subset G$, solve the problem $Z\left(f, G^{s}\right)$ with the help of the simplex-method, and find an optimal solution $x^{s}$.

3. If the obtained optimal solution is a permutation, i.e., a vertex of a permutable polyhedron $\Pi$, then check the fulfillment of constraints that have not been taken into account at the found point $x^{s}$. Obviously, such constraints can be only those describing a convex polyhedral set $D$. If the solution $x^{S}$ does not satisfy these (additional) constraints, then add the most violated constraint on the polyhedral set $D$ to the constraints of the admissible domain of the problem $Z\left(f, G^{S}\right)$. If the solution $x^{S}$ satisfies the mentioned constraints, then it is an efficient solution of the problem $Z(F, G)$ and, hence, of the problem $Z(F, X)$.

4. If the obtained solution $x^{s}$ is not a permutation, construct a cutting passing through adjacent vertices and cutting off a vertex that is not (a) feasible (permutation). Add this cutting to the constraints of the problem $Z\left(f, G^{s}\right)$.

5. Compare the value of $f\left(x^{s}\right)$ with the value of the objective function that is found at the previous step. If it decreases, then eliminate inactive (insignificant) constraints at the point $x^{s}$. If the value of $f\left(x^{s}\right)$ does not change, then do not eliminate any constraints. With the changed admissible domain of the problem $Z\left(f, G^{S}\right)$, pass to item 2 to solve this problem.

The circumstance that any constraint is not eliminated if $f\left(x^{s}\right)$ remains equal to the previous value guarantees the solution of only a finite number of problems of the form $Z\left(f, G^{S}\right)$.

The general idea of the proposed method consists of successive addition of the problem constraints that describe its domain of feasible solutions. A realization of the method in the form of an algorithm is described below.

To check the membership of a point in a set of permutations $P_{n k}(A)$, it makes sense to use the theorems $[9,10]$ formulated below.

THEOREM 6. If $x=\left(x_{1}, x_{2}, \ldots, x_{n}\right)$ is a point whose coordinates are ordered as follows: $x_{\alpha_{j}} \leq x_{\alpha_{j+1}} \forall j \in N_{n-1}$ and the following constraint is satisfied:

$$
x_{\alpha_{1}}+x_{\alpha_{2}}+\ldots+x_{\alpha_{i}} \geq a_{1}+a_{2}+\ldots+a_{i}
$$

that belongs to the ith group of inequalities of system (7), (8), then all the other inequalities of the ith group of this system are also satisfied at the point $x$.

THEOREM 7. A point $x=\left(x_{1}, x_{2}, \ldots, x_{n}\right)$ is a vertex of a permutable polyhedron $\Pi_{n k}(A)$ if and only if the following conditions are satisfied:

$$
\left\{\alpha_{1}^{1}\right\} \subset\left\{\alpha_{1}^{2}, \alpha_{2}^{2}\right\} \subset \ldots \subset\left\{\alpha_{1}^{n-1}, \ldots, \alpha_{n-1}^{n-1}\right\} \subset\left\{\alpha_{1}^{n}, \ldots, \alpha_{n}^{n}\right\}=N_{n}, \quad \sum_{t=1}^{i} x_{\alpha_{t}^{i}}=\sum_{t=1}^{i} a_{t} \quad \forall i \in N_{n} .
$$

During the realization of the algorithm for solving the problem $Z(F, X)$, the above theorems allow one to minimize the time required to check the found point for the satisfaction of combinatorial constraints and to reduce the number of constraints in the initial system.

The proposed algorithm can be used to solve the problem $Z(F, G)$ without taking into account all its constraints.

We pass to the presentation of the algorithm.

\section{ALGORITHM OF SOLUTION OF MULTICRITERIA COMBINATORIAL PROBLEMS OVER PERMUTATIONS}

\section{Initial step}

Put $s=0$. Reduce the multicriteria combinatorial problem $Z(F, G)$ to a one-criterion problem with the help of linear convolution as follows: we specify nonnegative weight coefficients $\lambda_{j}, j \in N_{l}$, that determine the degree of significance of each criterion and maximize the linear combination of objective functions, i.e., solve the problem

$$
Z\left(f, G^{s}\right):\left\{\max f(x)=\sum_{i=1}^{l} \lambda_{i}\left\langle c_{i}, x\right\rangle \mid \lambda_{i} \geq 0, i \in N_{l}, \sum_{i=1}^{l} \lambda_{i}=1, x \in G^{s}\right\} .
$$


If any coefficient $\lambda_{i}=1$ and all the other $\lambda_{j}=0, i \neq j, i, j \in N_{l}$, then consider a one-criterion problem with the $i$ th objective function. During the first execution of this step, we put $G^{S}=R^{n}$ and $\bar{f}=\infty$.

\section{Main part}

1. Choose an arbitrary initial point $x^{s}$ as an element of the general set of permutations or, according to Statement 2, order coefficients $\lambda_{i} \cdot c_{i}, i \in N_{l}$, of the objective function $f(x)=\sum_{i=1}^{l} \lambda_{i}\left\langle c_{i}, x\right\rangle$ and compute the value of $\bar{f}=f\left(x^{s}\right)$.

2. Describe the constraints that correspond to the point $x^{S}$ and determine a vertex of a general permutable polyhedron $\Pi_{n k}(A), x^{s} \in \Pi_{n k}^{s}(A), \Pi_{n k}^{s}(A) \supset \Pi_{n k}(A)$. Put $Q_{s}=N_{p}$.

3. Find deviations $r_{i}\left(x^{s}\right) \forall i \in R_{s}=N_{q} \backslash Q_{s}$ by formulas (19), (20).

4. Choose $r\left(x^{s}\right)=\max \left\{r_{i}\left(x^{s}\right) \mid i \in R_{s}\right\}$ and a number $i_{s} \in R_{s}$ for which the maximum is obtained.

5. Check the inequality $r\left(x^{s}\right) \leq 0$. If the inequality $r\left(x^{s}\right)>0$ is true, then pass to the following item of the algorithm and, otherwise, find an efficient solution of the problem $Z(F, X)$. To find the next efficient solution, pass to the initial step of the algorithm after assigning other weight coefficients $\lambda_{j}, \lambda_{j} \geq 0, j \in N_{l}$, and $\sum_{j=1}^{l} \lambda_{i}=1$.

6. Add the obtained constraint whose number is $i_{S} \in R_{S}$ to the constraints of the problem $Z\left(f, G^{s}\right)$, i.e., form the feasible set of the subproblem $Z\left(f, G^{s}\right)$ as follows:

$$
G^{s+1}=G^{s} \cap\left\{x \in R^{n} \mid\left\langle h_{i_{s}}, x\right\rangle \leq g_{i_{s}}\right\}
$$

7. If the following inequality is true:

$$
f\left(x^{s}\right)<\bar{f},
$$

then determine a set $N^{n}\left(x^{s}\right) \subset Q_{s}$, replace the set $Q_{s}$ by $Q_{s} \backslash N^{n}\left(x^{s}\right)$, and put $\bar{f}=f\left(x^{s}\right), s=s+1$.

8. Solve the problem

$$
Z\left(f, G^{s}\right): \max \left\{f(x)=\sum_{i=1}^{l} \lambda_{i}\left\langle c_{i}, x\right\rangle \mid x \in G^{s}\right\}
$$

by the dual simplex-method. If this problem has no solution, then the problem $Z(F, G)$ is also undecidable. Otherwise, we obtain an optimal solution $x^{s}$ of this problem. If it is not an element of the general set of permutations $P_{n k}(A)$, then pass to item 8. Otherwise, put $Q_{s}=N_{p}$ and pass to item 3 of the algorithm.

9. Find the vertices of the permutable polyhedron that are adjacent to the point $x^{s}$ and construct the cutting that passes through these vertices, is of the form

$$
\left\langle h_{i}, x\right\rangle \leq g_{i},
$$

and is such that the obtained point $x^{s}$ does not satisfy it. Form the system of constraints describing the set $G^{S}$ by formula (21) and pass to item 7.

Comment 1 (on item 7 of the algorithm). If $x^{s}$ is an element of the general set of permutations $P_{n k}(A)$, then all the constraints on the polyhedron $\Pi_{n k}(A)$ except for those determining the point $x^{s}$ should be assigned to the set $N^{n}\left(x^{s}\right)$ of inactive constraints that are eliminated from the set $Q_{s}$.

Comment 2 (to item 9 of the algorithm). Let us consider the determination of an adjacent vertex and construction of a cutting in more detail.

According to the theory of linear programming [15], based on the simplex table that determines some vertex $x^{s}$ of the polyhedron of solutions, a vertex adjacent to it can be obtained as a result of choosing a nonbasic variable $x_{j}$ in the linear programming problem in which the $j$ th vector $P_{j}$ has at least one positive component and choosing the $t$ th string of the simplex table under the condition

$$
\frac{g_{t}}{h_{i j}}=\min _{i: h_{i j}>0} \frac{g_{i}}{h_{i j}}=\Theta_{j},
$$

where $h_{i j}$ are coefficients multiplying unknowns $x_{j}$ in the $i$ th row and $g_{i}$ is a free term in the corresponding constraints of the linear programming problem $Z(f, G)$. Then the vector $P_{j}$ should be introduced into the basis instead of $P_{t}$ to obtain the simplex table for some vertex adjacent to $x^{s}$. 
Construction of cuttings [11]. We denote by $J$ the collection of numbers of nonbasic variables for which relationship (24) can be specified and by $I$ the set of numbers of basic variables. Based on the latter simplex table of the linear programming problem, we write the following constraint that is determined by a basic variable (with a number $i$ ):

$$
x_{i}+h_{i,(\beta+1)} x_{j_{1}}+\ldots+h_{i,(\beta+\gamma)} x_{j_{\gamma}}=g_{i},
$$

where $i \in I, \beta=|I|, \gamma=|J|, I \cup J=N_{n+1}, \beta+\gamma=n+1$, and $j_{\tau} \in J \forall \tau \in N_{\gamma}$.

Let $x^{*}=\left(x_{1}^{*}, \ldots, x_{n}^{*}\right)$ be an optimal solution (of the linear programming problem) to which corresponds the last simplex table. As is well known [15], if $j_{\tau}\left(j_{\tau} \in J \forall \tau \in N\right)$ are numbers of nonbasic variables in the solution of the linear programming problem $Z\left(f, G^{S}\right)$ and the value of $\Theta_{j}$ is computed by formula (24), all the vertices of the admissible domain that are adjacent to $x^{*}$ satisfy the inequality

$$
\frac{x_{j_{1}}}{\Theta_{j_{1}}}+\frac{x_{j_{2}}}{\Theta_{j_{2}}}+\ldots+\frac{x_{j_{\gamma}}}{\Theta_{j_{\gamma}}} \geq 1
$$

as the equality, and the point $x^{*}$ does not satisfy inequality (25).

According to the aforesaid, we construct cutting (23) in the form (25). The hyperplane forming the boundary of cutting (25) passes through adjacent vertices to the point $x^{*}$ being cut. The constructed hyperplane intersects facets of the admissible domain $G^{S}$ only at its vertices. Thus, new vertices are not created in the domain and the number of vertices of the domain is decremented by one.

THEOREM 8. The algorithm halts after solving a finite number of subproblems $Z\left(f, G^{s}\right)$ and produces an efficient solution of the problem $Z(F, X)$ or constructs a set of constraints that is such that the current subproblem $Z\left(f, G^{S}\right)$ is undecidable.

Proof. Since $X$ is a finite set, it has a finite number of subsets. With the step-by-step decrease in the value of the objective function $f(x)=\max \sum_{i=1}^{l} \lambda_{i}\left\langle c_{i}, x\right\rangle$, any subset cannot be repeated. Since any constraint is not rejected if we have $f\left(x^{s}\right)=\bar{f}$ and at least one or two constraints are added, the values of $f\left(x^{s}\right)$ can remain constant only during a finite number of iterations. Thus, the procedure comes to an end after a finite number of steps.

We note that the method of solution of a problem over permutations does not answer the question whether the solution found is strictly efficient. This can be checked using relationship (17).

\section{MATHEMATICAL MODELS OF SOME APPLIED PROBLEMS}

Problem 1 (maximization of information rate and mapping quality). Let us consider a system that accumulates information obtained from object domains (portals) and transfers it to personal computers (servers, workstations, terminals, etc.). We have $m$ object domains $A_{i}, i \in N_{m}$, in each of which a definite number of information units $a_{i}^{k}$ of the $k$ th form, $k \in N_{p}, a_{i}^{k} \in A$, are accumulated, i.e., $a_{i}^{k}$ is an element of a multiset $A=\left\{a_{1}, \ldots, a_{s}\right\}, s=m p$. This information is distributed among $n$ personal computers $B_{j}, j \in N_{n}$, on each of which no less than $b_{j}^{k}$ information units of the $k$ th form from some object domain are allocated. The transmission rate of the information unit of the $k$ th type from $A_{i}, i \in N_{m}$, of a definite object domain on personal computers $B_{j}, j \in N_{n}$, equals $\theta_{i j}^{k}$, and the quality coefficient of representation of the information unit of the $k$ th type from a definite object domain $A_{i}, i \in N_{m}$, equals $d_{i j}^{k}$ under the condition of its quality representation on personal computers $B_{j}, j \in N_{n}$.

It is necessary to determine a plan of transmitting and loading an amount $x_{i j}^{k}$ of information of the $k$ th form, $k \in N_{p}$, from object domains $A_{i}, i \in N_{m}$, to personal computers $B_{j}, j \in N_{n}$, so that the total transmission rates are maximal and the total quality load factor is maximized.

Mathematical model. It is required to determine

$$
f_{1}(x)=\max \sum_{k=1}^{p} \sum_{i=1}^{m} \sum_{j=1}^{n} \theta_{i j}^{k} x_{i j}^{k}, \quad f_{2}(x)=\max \sum_{k=1}^{p} \sum_{i=1}^{m} \sum_{j=1}^{n} d_{i j}^{k} x_{i j}^{k}
$$

under combinatorial conditions that take into account permutable properties of the domain of feasible solutions of the 
following problem:

$$
x=\left(x_{11}^{1}, \ldots, x_{1 n}^{1}, \ldots, x_{1 p}^{p}, \ldots, x_{m p}^{p}\right)=\left(x_{1}, \ldots, x_{s}\right) \in P_{s \eta}(A)
$$

and constraints on amounts of information of definite type that are loaded into each personal computer $\sum_{i=1}^{m} x_{i j}^{k} \leq b_{j}^{k}$, $j \in N_{n}, k \in N_{p}$.

Problem 2 (computations on a supercomputer). Let us consider a cluster supercomputer that performs highly intensive parallel computations using definite programs. We consider that the main characteristics of the cluster are its processor throughput and main memory. To process their data, definite programs require appropriate main memory sizes and computational speeds. These programs should be distributed among clusters so that the total performance and workload of the cluster supercomputer are maximal and each program is executed on an individual cluster.

Mathematical model. Let a cluster supercomputer consist of $m$ clusters $A_{i}$ each of which is characterized by its main memory size or its performance (clock frequency) $a_{i}, i \in N_{m}$. It is required to simultaneously load $n$ program complexes $B_{j}$, $j \in N_{n}$, to be performed each of which is characterized by its main memory size or computational speed $b_{j}$.

The performance $p_{i j}^{k}, i \in N_{m}, j \in N_{n}$, of the cluster supercomputer is determined by the number of operations performed per second by each processor of the corresponding clusters in executing the $k$ th program of the $j$ th program complex.

We call occupied the $i$ th cluster that is $100 \%$ loaded during the execution of the $k$ th program of the $j$ th program complex. Then the workload is defined as the difference $d_{i j}^{k}=100-d_{i j}^{3}$, where $d_{i j}^{3}$ is the current cluster workload (in percentage terms) during the execution of the $k$ th program of the $j$ th program complex on the $i$ th cluster, $i \in N_{m}, j \in N_{n}$.

A variable $x_{i j}^{k}, k \in N_{p}, i \in N_{m}, j \in N_{n}$, characterizes the main memory size required for the execution of the $k$ th program of the $j$ th program complex on the $i$ th cluster and can assume any value from a multiset $A$, where $A=\left\{a_{1}, \ldots, a_{S}\right\}$, $s=p m n ; P_{s \eta}(A)$ is the general set of permutations of all elements from the multiset $A$, where $\eta$ is the number of different elements in $A$. We note that some elements from $A$ can be zero.

The mathematical model of the problem can be described as follows:

maximize the computer workload

$$
f_{1}(x)=\max \sum_{k=1}^{p} \sum_{i=1}^{m} \sum_{j=1}^{n} d_{i j}^{k} x_{i j}^{k}
$$

and its performance

$$
f_{2}(x)=\max \sum_{k=1}^{p} \sum_{i=1}^{m} \sum_{j=1}^{n} p_{i j}^{k} x_{i j}^{k}
$$

under constraints on the main memory sizes of loaded programs for each program complex and each cluster $\sum_{i=1}^{m} \sum_{k=1}^{p} x_{i j}^{k} \leq b_{j}, j \in N_{n}, \sum_{j=1}^{n} \sum_{k=1}^{p} x_{i j}^{k} \leq a_{i}, i \in N_{m}$.

This model provides the maximal use of resources of the cluster supercomputer.

Example. Let an operating cluster supercomputer for parallel computations consist of three clusters with definite characteristics (Table 1). It is necessary to simultaneously load three programs to be executed that require 80,35 , and 95 GB of main memory.

Solution. The possible workload of clusters will be $80 \%, 60 \%$, and $50 \%$, respectively. We compose the model of the problem in the form

$$
\begin{gathered}
\max F_{1}=4.2 x_{1}+2.2 x_{2}+1.2 x_{3}, \\
\max F_{2}=0.8 x_{1}+0.6 x_{2}+0.5 x_{3}, \\
x_{1}+x_{2}+x_{3} \leq 100+150+70 .
\end{gathered}
$$

Let us consider the set $A=\{35 ; 80 ; 95\}$. It is obvious that the permutation $x=\left(x_{1}, x_{2}, x_{3}\right)=(95,80,35)$ is an optimal solution of the problem with the partial criterion values equal to

$$
\max F_{1}=4.2 \cdot 95+2.2 \cdot 80+1.2 \cdot 35=617, \quad \max F_{2}=0.8 \cdot 95+0.6 \cdot 80+0.5 \cdot 35=141.5 .
$$


TABLE 1

\begin{tabular}{|c|c|c|c|}
\hline $\begin{array}{c}\text { Number of } \\
\text { a cluster }\end{array}$ & Main memory, GB & Performance, GFLOP/sec & $\begin{array}{c}\text { Cluster workload for } \\
\text { the current period, \% }\end{array}$ \\
\hline 1 & 100 & 4.2 & 20 \\
2 & 150 & 2.2 & 40 \\
3 & 70 & 1.2 & 50 \\
\hline
\end{tabular}

\section{CONCLUSIONS}

In the present article, complicated combinatorial multicriteria problems over a set of permutations are investigated. Some properties of the admissible domain of a combinatorial multicriteria problem embedded into an arithmetic Euclidean space are considered. A method of solution of the formulated problems is constructed and proved. The approach proposed can be used for solution of multicriteria problems over combinatorial sets of permutations. A software implementation of this approach gives the possibility to investigate and to find the elements of sets of Pareto-optimal solutions for multicriteria combinatorial problems with allowance made for other combinatorial properties of the domain of feasible solutions.

The further development of this work will be directed toward the realization and adaptation of the proposed algorithm and also toward the development of new methods for solution of vector combinatorial optimization problems.

\section{REFERENCES}

1. I. V. Sergienko, Mathematical Models and Methods for Solving Discrete Optimization Problems [in Russian], Naukova Dumka, Kiev (1988).

2. I. V. Sergienko and M. F. Kaspshitskaya, Models and Methods for the Computer Solution of Combinatorial Optimization Problems [in Russian], Naukova Dumka, Kiev (1981).

3. I. V. Sergienko, L. N. Kozeratskaya, and T. T. Lebedeva, Stability Investigation and Parametric Analysis of Discrete Optimization Problems [in Russian], Naukova Dumka, Kiev (1995).

4. I. V. Sergienko, T. T. Lebedeva, and N. V. Semenova, "Existence of solutions in vector optimization problems," Cybernetics and Systems Analysis, No. 6, 39-46 (2000).

5. T. T. Lebedeva, N. V. Semenova, and T. I. Sergienko, "Optimality and decidability conditions of in linear vector optimization problems with a convex admissible set," Dop. NAN Ukr., No. 10, 80-85 (2003).

6. I. V. Sergienko, V. A. Roshchin, and N. V. Semenova, "Some integer programming problems with ambiguous data and their solution," Probl. Upravlen. Inf., No. 6, 116-123 (1998).

7. T. T. Lebedeva, N. V. Semenova, and T. I. Sergienko, "Stability of vector problems of integer optimization: Relationship with the stability of sets of optimal and nonoptimal solutions," Cybernetics and Systems Analysis, No. 4 , 90-100 (2005).

8. V. V. Podinovskii and V. D. Nogin, Pareto-Optimal Solutions of Multicriteria Problems [in Russian], Nauka, Moscow (1982).

9. Yu. G. Stoyan and S. V. Yakovlev, Mathematical Models and Optimization Methods of Geometric Design [in Russian], Naukova Dumka, Kiev (1986).

10. Yu. G. Stoyan and O. O. Yemets, Theory and Methods of Euclidean Combinatorial Optimization [in Ukrainian], Inst. Sist. Doslid. Osvity, Kyiv (1993).

11. O. O. Yemets and L. M. Kolechkina, Combinatory Optimization Problems with Linear-Fractional Objective Functions [in Ukrainian], Naukova Dumka, Kyiv (2005).

12. V. A. Roshchin, N. V. Semenova, and I. V. Sergienko, "Solution and investigation of one class of inexact integer programming problems," Cybernetics, No. 2, 42-47 (1989).

13. V. A. Roshchin, N. V. Semenova, and I. V. Sergienko, "Decomposition approach to solving some integer programming problems with inexact data," Zh. Vychisl. Mat. Mat. Fiz., 29, No. 5, 786-791 (1990).

14. L. S. Lasdon, Large-Scale Systems Optimization [Russian translation], Mir, Moscow (1975).

15. Yu. M. Ermol'ev, I. I. Lyashko, V. S. Mikhalevich, and V. I. Tyuptya, Mathematical Methods of Operations Research [in Russian], Vyshcha Shkola, Kiev (1979). 